\title{
In Memoriam: Andrei Suslin
}

\section{Eric M. Friedlander and Alexander Merkurjev}

We present a collection of reminiscences about the life and mathematical contributions of our friend Andrei Suslin, who died in July 2018 at the age of sixty-seven. Andrei was an exceptionally gifted mathematician and a very special person. Unlike most outstanding Russian mathematicians, Andrei attended an English language school (in Leningrad, now St. Petersburg) rather than a specialized mathematics school. At the age of thirteen, he was awarded first prize at the Leningrad Math Olympiad and continued to win first prize at this student competition for several more years. At the age of seventeen, Andrei received a gold medal at the International Math Olympiad. That year, 1968, Andrei was diagnosed with diabetes, ending his successful gymnastics career and his participation in summer sports camps. At Leningrad State University, Andrei was formally a student of Mark Bashmakov, although Andrei studied independently by reading Bourbaki as well as works by J.-P. Serre. Andrei's individualism was reflected by his avoidance of politics during those Soviet times. For example, as the youngest doctor of sciences in the Soviet Union, Andrei was offered membership in the Central Committee of the Young Communist League (Comsomol), which he declined despite the accompanying offer of an apartment of his own.

Every summer during his early career as a student and then as a faculty member, Andrei led a team of friends (including other mathematicians) who built houses (dachas) outside of the city of Leningrad. This was an activity he enjoyed. He was a skilled carpenter, and the houses built by his team were in considerable demand. Nevertheless,

Eric M. Friedlander is Dean's Professor of Mathematics at the University of Southern California. His email address is ericmf@usc. edu.

Alexander Merkurjev is a professor of mathematics at the University of California, Los Angeles. His email address is merkurev@math. ucla.edu.

For permission to reprint this article, please contact:

reprint-permission@ams.org.

DOI: https://doi.org/10.1090/noti2101

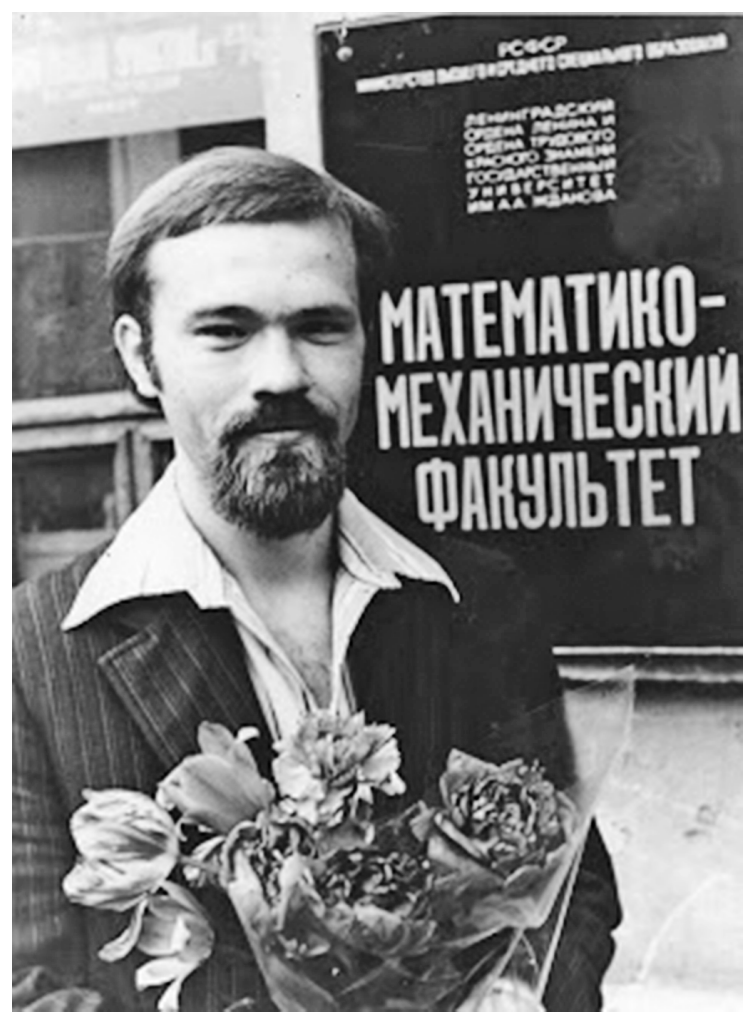

Figure 1. Suslin after defense of second doctoral thesis. Leningrad, 1977.

when local residents learned that Andrei was a professor of mathematics, they lowered his salary.

Andrei burst onto the international mathematical scene with his proof of Serre's conjecture in 1976. This result, the most famous conjecture at the time in commutative algebra, affirmed that every projective module over a ring $R$ is a free module, provided the ring $R$ is a polynomial ring over a field. This led to Andrei's first invited lecture at the International Congress of Mathematicians (ICM) at Helsinki in 1978.

Unfortunately, Andrei was not permitted to travel to the United States to give his second ICM talk, a plenary talk at the ICM at Berkeley in 1986. Instead, Eric Friedlander presented Andrei's work based upon Andrei's manuscript 


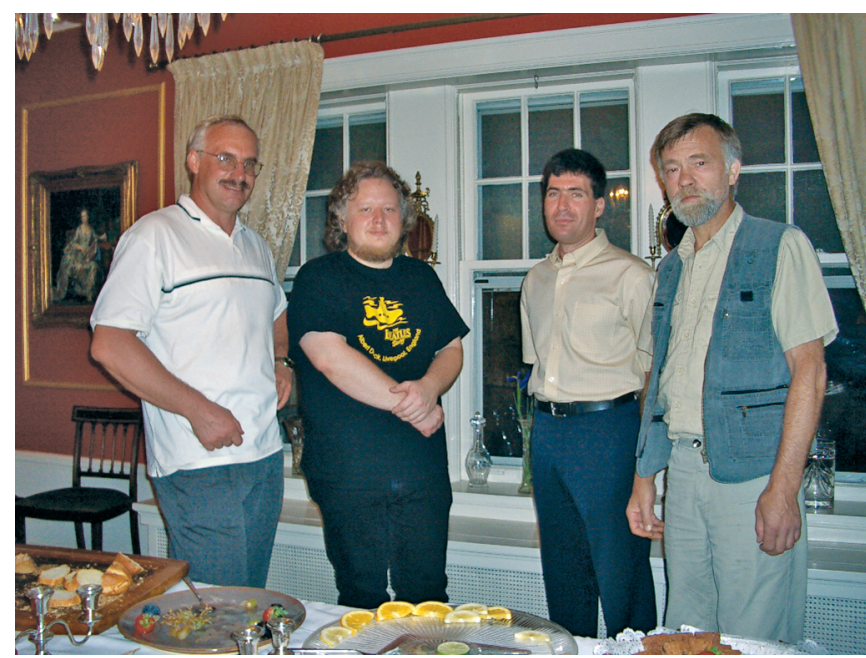

Figure 2. At the Friedlanders' house, with his graduate students Seva Joukhovitski and Serge Yagunov, 2004.

with theorem after theorem about the algebraic $K$-theory of commutative rings, especially the algebraic $K$-theory of fields. These results include techniques, computations, and a general vision which have guided the subject. The most dramatic of these results was the theorem of Merkurjev and Suslin relating algebraic $K_{2}$ to étale cohomology (extended to $K_{3}$ modulo 2), the subject of Merkurjev's invited talk at the same Berkeley ICM. Another highlight was Andrei's proof of the Quillen-Lichtenbaum conjecture for algebraically closed fields, which introduced transfers for correspondences. Another was Andrei's theorem relating Milnor $K$-theory to algebraic $K$-theory.

Andrei provided definitive results concerning many well-known problems, including his proof with Wodzicki of the Karoubi Problem on excision in the $K$-theory of $C^{*}$ algebras.

His many results about homological stability, central simple algebras, and Severi-Brauer varieties have paved the way to much new research.

Andrei gave his third invited ICM talk at Zurich in 1994. This lecture focused on motivic cohomology, a theory developed by Suslin and Voevodsky which has significantly influenced current research in algebraic geometry. Beginning with the Suslin complex, Andrei, in collaboration with Voevodsky, established the foundations of this theory, which has its origins in $K$-theory and étale cohomology.

Four years later, Friedlander gave an invited ICM talk in Berlin about joint work with Andrei concerning finite group schemes, work that once again led to many interesting developments (this time in representation theory). In particular, not only did Andrei play the major role in proving finite generation of cohomology, he also introduced strict polynomial functors and the geometry of 1-parameter subgroups. In 2000, Andrei received the AMS Cole Prize for his achievements.

It seems fair to say that Andrei's ability to confront and solve long-standing problems in algebra using ingenious techniques and difficult computations had no equal. Those of us who worked with Andrei and many others whose work intersected with Andrei's all appreciated his generosity, valued his guidance, and sought his seal of approval. In his quiet way, Andrei led the many successful efforts to understand the web of relationships between algebraic geometry, algebraic $K$-theory, algebraic number theory, and cohomology of groups.

What follows are personal reflections about Andrei's career as one of the most influential algebraists of the late twentieth century. The interested reader can consult our recent article in the AMS Bulletin giving an overview of some of Andrei Suslin's mathematical achievements.

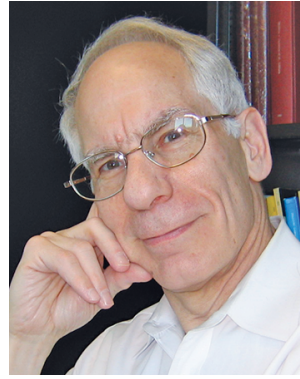

Eric M. Friedlander

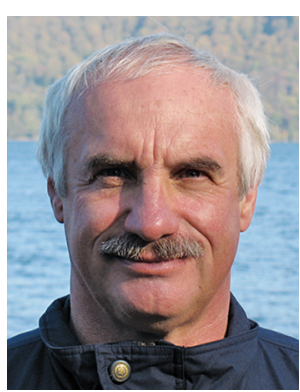

Alexander Merkurjev

\section{Alexander Beilinson}

In the 1980s Andrei used to come to Moscow from time to time. Listening to his talks at seminars one kept feeling that Andrei's way of thought comes from a different dimension, his arguments being called on by a magic whistle from the land where all perfect proofs live. I especially remember Andrei's explanation that Milnor's $K$-theory is naturally a quotient of Quillen's $K$-theory, the amazing rigidity argument he used to compute $K$-theory with finite coefficients of an algebraically closed field, and, of course, the majestic Merkurjev-Suslin theorem. And there was a talk where Andrei introduced the notion of finite correspondence and defined Suslin's homology. It was so clear that these were the right concepts to see the motivic cohomology; the only essential obstacle to overcome was to understand the compatibility with Zariski's localization. At the moment I had little doubt that Andrei would find a solution in a short while-but it came only in about nine years

Alexander Beilinson is David and Mary Winton Green University Professor at the University of Chicago. His email address is sasha@math. uchicago.edu. 
with Voevodsky bringing the Nisnevich topology into the game.

Looking back at these years I feel how happy they wereand Andrei's wonderful gift and his artist's freedom being a part of that luminous joy.

In the middle of the 1990s the motivic dreams became a reality due mostly to the effort of Volodya and Andrei. I learned the subject mostly from Andrei's great lectures at Northwestern around 2000. The first half of the course covered the formalism of Voevodsky's triangulated category of motives (skipping the proof of compatibility of the Nisnevich and $\mathbb{A}^{1}$-homotopy localizations); the second half treated Voevodsky's proof of the Milnor conjecture. The clarity and elegance of Andrei's exposition matched perfectly the beauty of mathematics he was explaining to us.

I miss you, Andrei.

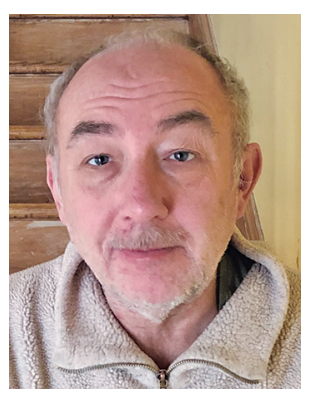

Alexander

Beilinson

\section{Eric M. Friedlander}

Andrei Suslin was a great mathematician. He was also my closest mathematical friend, someone I admired tremendously.

I was very fortunate to share mathematics with Andrei. What Andrei sought were explicit, difficult problems which could challenge him. Time and again, I was confronted by such a problem and Andrei alone could find a solution.

We usually worked in parallel: one common method of collaboration was for me to stare at the blackboard in my office at some mathematics I did not understand while Andrei paced outside, smoking cigarettes. Andrei never seemed to make mistakes, always seemed to fully understand all mathematics he encountered. Of course he was a powerful mathematician, but in addition to his evident talent he had amazing insights and an ability to totally focus.

For me, Andrei was much more than a brilliant mathematician with whom I participated in a small portion of his remarkable mathematical achievements. We were close friends, first meeting in the 1970s and colleagues for more than twenty years. There remain good memories of time together in Paris and in Evanston, dinners and drinking, skiing and talking. Despite our completely different backgrounds, we could communicate easily, often without words.

Andrei's mathematics will be eternal. His direct, generous, and kind personality will be treasured by his family and his friends.

\section{Christian Haesemeyer}

My first contact with Andrei Suslin was through a piece of his mathematics. While an undergraduate student in Bonn (it must have been in 1997), fascinated by both arithmetic algebraic geometry and algebraic topology, I came across his beautiful 1996 paper with V. Voevodsky on "Singular homology of abstract algebraic varieties" - here was a unificiation of these two themes! I was unaware at the time that Andrei had then already contributed revolutionary work in algebra and algebraic $K$-theory for thirty years. (There is no need to repeat his many brilliant achievements here, but Andrei's contribution is towering. It is difficult to come across major work in $K$-theory, for example, that hasn't been influenced by him-listing all the papers featuring a sentence like "our argument follows in its basic outlines that of Suslin in..." would take a lot of space in itself.) Learning more about algebraic cycles, motivic cohomology, and algebraic $K$-theory, I decided I wanted to do my PhD at Northwestern University, then home of Andrei Suslin, Vladimir Voevodsky, and Eric Friedlander. In the two years between my coming across that paper and my arrival at Northwestern, Andrei completed several more major pieces of work, both in the theory of motivic cohomology (including his massively influential paper with Voevodsky on the "Bloch-Kato conjecture and motivic cohomology with finite coefficients" in 1998) and in the study of group schemes and their cohomology (his joint work with Friedlander proving finite generation of cohomology for finite group schemes as well as his work with C. Bendel and Friedlander on infinitesimal group schemes and their support varieties all appeared in 1997). Further major pieces of work include his foundational book on the theory of cycles and their cohomology with Friedlander and Voevodsky in 2000 and his papers on the motivic spectral sequence with Friedlander in 2002 and his singly authored sequel in 2003.

I joined the PhD program at Northwestern University in the fall of 1999, and Andrei was gracious enough to accept me as a student almost immediately-I had the enormous privilege of advice from and discussion with both him and

Christian Haesemeyer is a professor of mathematics at the University of Melbourne. His email address is christian.haesemeyer@unime1b.edu.au. 
Eric Friedlander. Andrei's dedication to sharing mathematics was indeed one of his most outstanding qualities. For example, I remember that soon after he was scheduled to teach a course in homological algebra, and since about half the students enrolled already had a background in the basics of the subject, he decided he would simply teach two versions of it-the basic and advanced versions, thus doubling his teaching duties. He was equally free with his time in a one-on-one setting. While we didn't have the weekly student-advisor meetings typical of US PhD supervision (partly due to our rather different schedules, me being more of a morning person), our somewhat less regular meetings would usually go on for many hours, Andrei patiently listening to what I realized later-mostly just after the meetings ended-was often pretty much nonsense, while giving hints as to what path might be more promising. In addition to mathematics, I learned from Andrei how much can be explained in a one-hour lecture by a lecturer who has a really deep understanding of the material. Notes from any of his lectures or courses are something to be treasured; I have preserved mine through half a dozen moves. While famously not particularly interested in the "professional" parts of our profession, I also learned one crucial professional lesson from Andrei, grounded I believe in his experiences in the Soviet Union: that one should put the person before the institution.

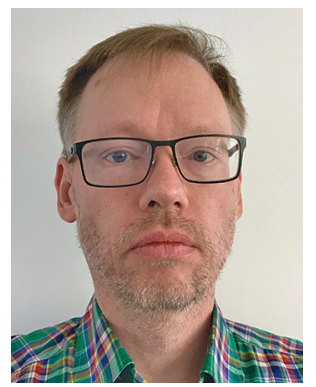

Christian

Haesemeyer

\section{Marc Levine}

Besides being a fantastic mathematician, I remember Andrei Suslin's wonderful warm-heartedness and generosity. At some early point in my career, I had twice proven results which I had really struggled with to bring to completion and was really proud of. Both times, Andrei came out with much more elegant solutions to the same problem at roughly the same time. This was before the fall of the Soviet Union, and sometime later, when I finally got to meet Andrei, I mentioned this to him, and his reply has stuck

Marc Levine is a professor of mathematics at the University of Duisburg-Essen. His email address is marc. 1evine@uni-due.de.

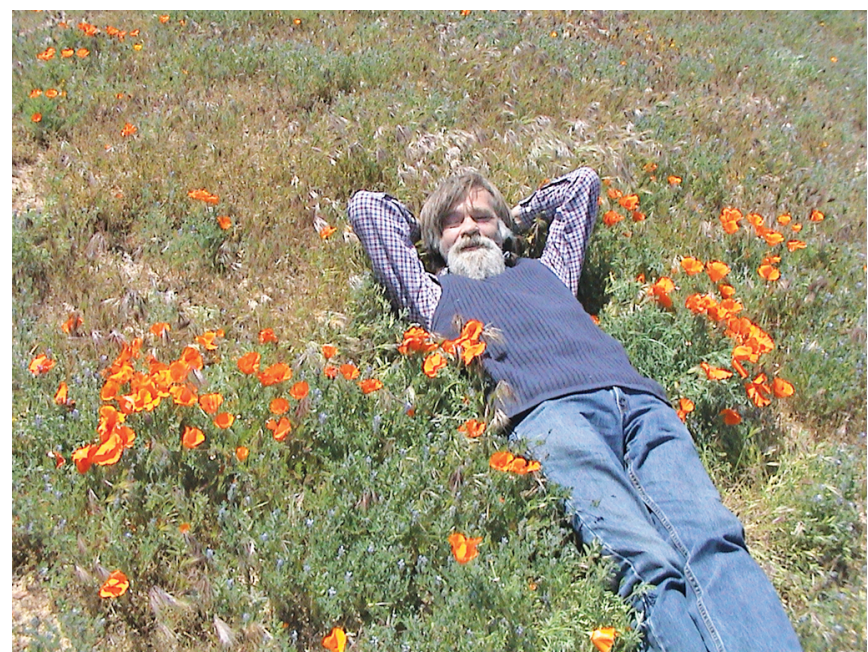

Figure 3. Antelope Valley, 2005.

with me to this day: "It's not a problem; you proved it first in the West, and I proved it first in the East."

I loved attending his lectures. Andrei had such a concise way of lecturing, with every sentence completely clear and packed with meaning, moving the lecture forward in striking leaps. It made for a strenuous journey on the part of the listener, but the reward was always worth the effort.

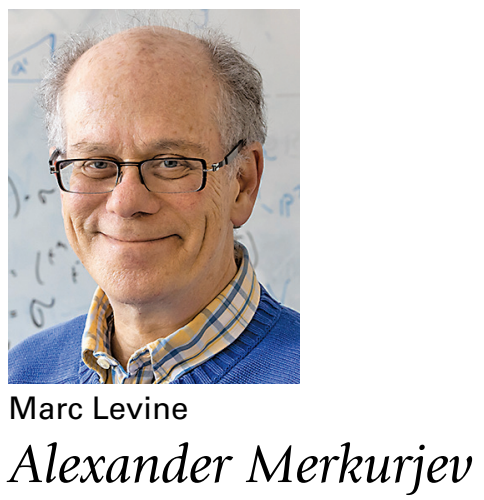

I first met Andrei Suslin in 1970. He was a third-year undergraduate student at the university, and at the same time he was my teacher at the high school which I attended. This Mathematics Boarding School \#45 was a unique, special place. It collected talented pupils in the northwest region of the Soviet Union. It was the only way into mathematics for many people living outside of big cities. Suslin taught at this school for three years. His style had a tremendous impact on me unlike any other experience. Needless to say that already at that time I decided to study algebra.

Andrei's passion for mathematics and his systematic approach were a model for us. We saw him reading algebra books like Bourbaki's commutative algebra in a bus or metro. During short breaks between lessons he would draw complicated diagrams. That time Andrei was 


\section{MEMORIAL TRIBUTE}

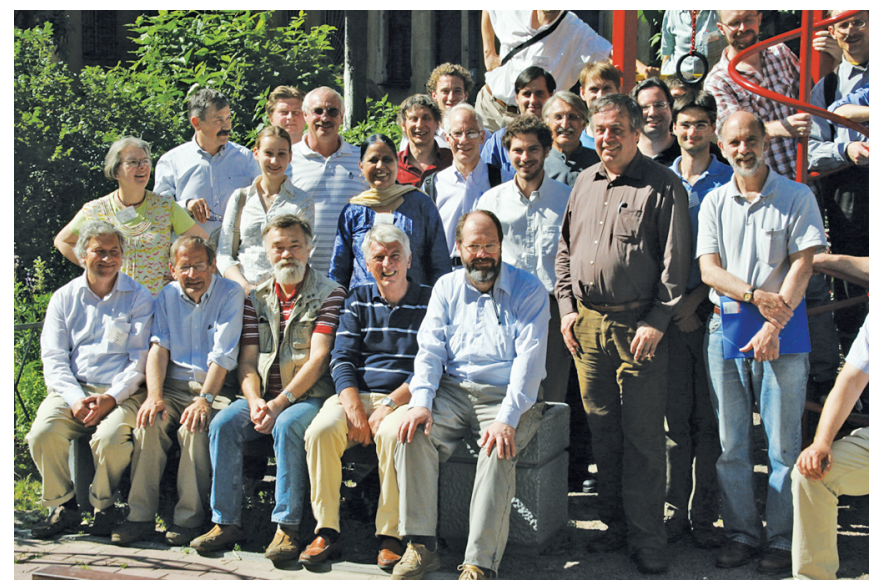

Figure 4. At the 2010 POMI, St. Petersburg conference in honor of Suslin.

working on a problem in finite geometry and combinatorics. I guess that work was not successful, and at the beginning of his senior year Andrei realized that he had nothing yet done for the diploma work which was supposed to be completed in nine months. That is how he turned to Serre's conjecture concerning modules over polynomial rings. His initial work was so successful that immediately after his graduation, Andrei was hired as an assistant professor at the university (he was therefore never a graduate student). Soon after, he solved Serre's conjecture.

Andrei was a problem solver. During boring meetings we had to sit through, Andrei would ask me to give him problems to solve from recent mathematical Olympiads, and often my list ended before the meeting was over. Andrei was a winner of the International Mathematical Olympiad in 1967.

His "Olympiad spirit" had an interesting consequence: Andrei considered every mathematical problem as a personal challenge. That is why there are not so many conjectures of Suslin's: by making a conjecture Andrei would have admitted that he failed to prove it himself.

Andrei's impact on mathematicians has been tremendous, not only on his own graduate students but on many others fortunate to be around him. I remember his spontaneous seminars, lasting for many hours. Andrei would start when people spontaneously would get together in his room at the Math Institute. I remember his talks on the foundations of motivic cohomology in the late 1980s, which were rather more like improvisations at the board than lectures. During these seminars Andrei generously shared his ideas.

We miss Andrei.

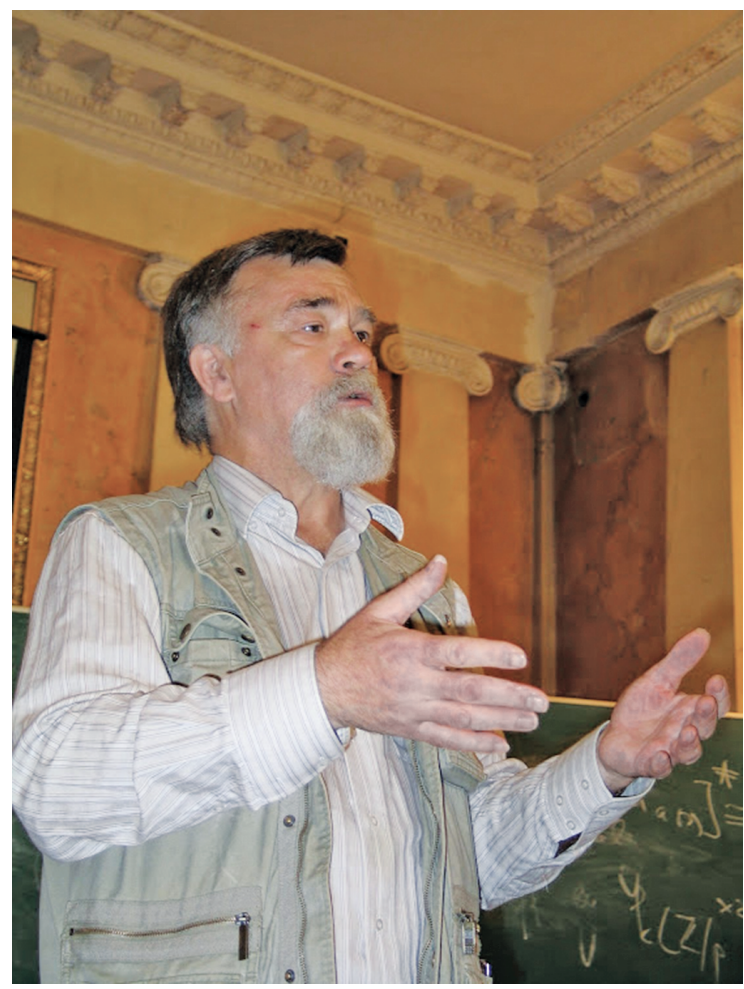

Figure 5. Suslin's talk at the same conference.

\section{Ivan Panin}

Suslin was my scientific advisor since my junior year at the university. Then he was my thesis advisor (1981-1984) at Steklov Mathematical Institute in Leningrad (now SanktPetersburg). But most importantly for me he was my mentor all my life. In this remembrance I simply want to recall a few episodes that most vividly characterize Suslin as a mathematician and a person.

In the 1990s I used to come to Suslin's office (room 306, Steklov Mathematical Institute) and ask him what he was working on. Suslin used to take a cup of tea and tell me what he was interested in. Then he started to explain his approach to the problem on the blackboard.

If something went wrong he took a cigarette or another cup of tea and after a short break offered another approach. Each time, before doing calculations, he formulated expected intermediate results and a scheme of the argument. And only after that he started to calculate, say, the first nontrivial case.

If something went wrong (this often happened), he again took a break, and all that repeated several times. Such discussion (I was mainly a listener) lasted for one, two, three hours. Several times they lasted for $4-5$ hours

Ivan Panin is a principal research fellow at the St. Petersburg Department of Steklov Institute of Mathematics. His email address is paniniv@gmai 1 . com. 
with some breaks when we drank tea with doughnuts. Due to such communications I learned a method that Suslin never explicitly formulated, but I am sure he systematically used it.

Let me explain this method since I think this is the most important thing that I learned from him (aside from the technicalities). First, using all previous experience, one formulates the statement to be proven. Then one needs to believe strongly that the statement is correct. And only after that one needs to look for a possible approach. As we know, in the course of solving difficult problems there arise technical (or substantial) complications so that we give up and lose interest in the problem.

We need to know a priori that the expected result is correct. I learned from Suslin this principle. Probably this is the most important method I learned from him.

Purely from a human point of view, because of Suslin, I was accepted into a wide circle of great mathematicians. The main principle of human relations I learned from Suslin was not to offend anyone. And he succeeded in doing so very well.

Now I would like to recall a particular interaction illustrating Suslin's taste in problems. We were drinking wine in his apartment in Evanston. "Vanya," asked Suslin, "what problem would you like to work on?" I gave some answer. Suslin said, "That is boring. Let's prove the Hodge conjecture or Grothendieck's standard conjecture." This was all Suslin. It was no fun for him to lift a stone, even a big one. A genuine challenge for him was to move a boulder.

For me this episode illustrates how he tried to solve very important problems.

In 1984 Suslin returned from IHES and happily said, "Vanya, I proved the Quillen-Lichtenbaum conjecture one night before my talk at IHES. Just after my talk, Gabber generalized my argument, and in a few days, using his generalization, I proved that $K_{i}^{Q}(\mathbb{C} ; \mathbb{Z} / n)=K_{i}^{\text {top }}(p t ; \mathbb{Z} / n)=$ $\mathbb{Z} / n$ if $i \geq 0$ is even and 0 otherwise. The stable version of the Milnor-Friedlander conjecture comes up as a corollary. Namely, $H^{*}(\mathrm{GL}(\mathbb{C}) ; \mathbb{Z} / n)$ is a polynomial ring $\mathbb{Z} / n\left[c_{1}, c_{2}, c_{3}, \ldots\right]$. In other words, cohomology with finite coefficients of the classifying space of the group $\mathrm{GL}(\mathbb{C})$, viewed as a discrete group, is the same as cohomology of the classifying space of the group $\mathrm{GL}(\mathbb{C})$, viewed as a Lie group." After this introduction, Suslin in half an hour sketched a proof of his celebrated rigidity theorem.

In 1999 at MPI in Bonn Suslin asked me and Serge Yagunov, "What are you working on now?" We answered that we wanted to move the rigidity theorem in $K$-theory to the Voevodsky cobordisms. To our surprise, Suslin became excited and discussed the problem with us for three days. On the third day he came and said, "All you need to prove is the following three properties of the Gysin homomorphisms:

(1) the base change property,

(2) covariant functoriality,

(3) normalization.

Having all these three properties, you will prove the rigidity." This episode vividly illustrates Suslin's style in mathematics.

In January of 1994, Suslin entered his office handling a preprint. "This is a brilliant work," he said. It was a handwritten copy of a celebrated preprint of Voevodsky on presheaves with transfers. Suslin immediately started to explain the paper and overall gave four lectures four hours each. This preprint formed the basis of Voevodsky's triangulated category of motives that finally led to the proof of Milnor's conjecture.

Sometime in 1995 I asked Suslin to explain Quillen's trick to me, which Quillen invented in the proof of Gersten's conjecture. Within five minutes Suslin spelled it out for me and formulated the following principle: in such problems one always needs to start with the closed fiber. The same holds for Voevodsky's first trick. In the twenty years that followed, my coauthors and I used this fundamental principle in the solution of many problems. During the following evening with Suslin, we proved the Grothendieck-Serre conjecture for the group $\mathrm{SL}_{1, A}$ where $A$ is an Azumaya algebra. Since then I started to work hard on the Grothendieck-Serre conjecture, and Suslin returned to the study of motivic cohomology with Voevodsky.

Let me stop here and simply say: I offer this in loving memory of my great teacher and of the great mathematician Andrei Suslin.

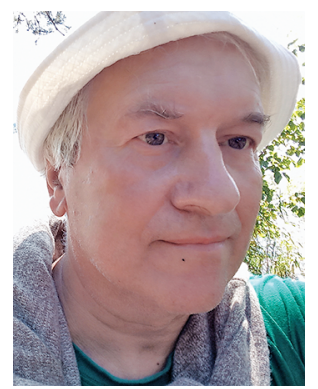

Ivan Panin

\section{Raman Parimala}

When I joined the Tata Institute (TIFR) in Mumbai in the early 1970s as a young researcher, the most exciting

Raman Parimala is the Arts \& Sciences Distinguished Professor of Mathematics at Emory University. Her email address is parima 1 a@mathcs . emory. edu. 
conversations one could overhear in the corridors of the third floor were on Serre's question on projective modules, namely, whether projective modules over polynomial rings $k\left[x_{1}, x_{2}, \ldots, x_{n}\right]$ for a field $k$ are actually free. When Serre's question was answered in the affirmative by Quillen and Suslin independently in 1976, there were literally celebrations on the third floor of the institute by the jubilant algebraists!

It was at the 1978 ICM held in Helsinki, Finland, that I first met Andrei Suslin in person. I was a postdoc at the ETH Zurich at that time. He had just given his spectacular invited address on the cancellation problem for projective modules. Amidst the congratulatory messages pouring in from eminent well-wishers and friends, I managed to introduce myself to him. He quietly turned towards me saying, "I have something that might be of interest to you, Parimala" and produced a LOMI preprint of his work with V. I. Kopeiko on the quadratic analogue of Serre's question, namely, whether quadratic spaces on the affine $n$ space over a field of characteristic not 2 are pullbacks of spaces from the field. I still remember the thrill that I felt on hearing that he had read my work! Indeed, Suslin's solution of Serre's question and further work on stability results for projective modules were a source of great inspiration and influence for the algebraists at Tata, heralding an era of beautiful work in this area of research.

I remember with delight a memorable series of lectures given by Suslin in 1981 when he was visiting TIFR on the famous Merkurjev-Suslin norm residue isomorphism theorem. This result established an isomorphism between the group $K_{2}(k) / n K_{2}(k)$, from the world of $K$-theory, and the group $H^{2}\left(k, \mu_{n}\right)$, from the world of Galois cohomology. In fact, the manuscript of the paper of Merkurjev-Suslin was typeset at the mathematics office of the TIFR, and a copy of the typescript was for a long time my source of reference to this article! While I enjoyed the startlingly beautiful algebra being presented, the true depth and significance of the result only dawned on me more than a decade later when I started working on a conjecture of Serre (Conjecture II) along with Eva Bayer. The conjecture for groups of inner type $A_{n}$ translates to the surjectivity of reduced norm for central division algebras over a perfect field of cohomological dimension 2. Merkurjev and Suslin prove the conjecture for this case. In fact, they gave a characterization of fields of cohomological dimension 2 in terms of reduced norm surjectivity for all finite extensions of the field-a key ingredient being the norm residue isomorphism theorem.

Apart from mathematics, during his visit to TIFR, Suslin had a penchant for playing volleyball during the thick Mumbai monsoon rains. One would typically find him enjoying the endless rain while hard at play at a game on the TIFR grounds. I also remember how he boldly embraced

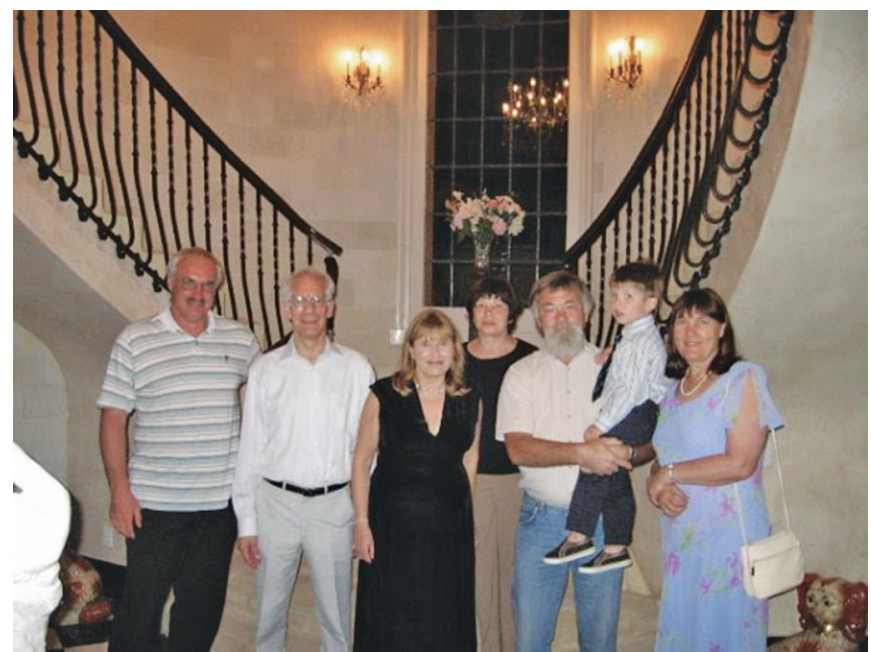

Figure 6. At Eric Friedlander's house, 2011.

the local cuisine and audaciously frequented the East Canteen known for its lavishness when it came to using Indian spices, while more cautious colleagues dined at the West Canteen, which was deemed more suitable for the nonIndian palate.

Another interaction with Suslin that stands out in my memory was at a very pleasant conference titled Algebra and Applications that took place in IIT Delhi (also in '81), where I was also scheduled to give a talk. It saw huge participation from mathematicians from all over India and also from overseas. Unfortunately, the speaker right before me overshot his time and was sternly reprimanded by the chair of his session, which unnerved me a little. Suslin, who was sitting beside me smilingly assured me, "Don't worry. I am the chair for your session. You can speak for a couple of hours and I won't interrupt!"

I have had the pleasure of listening to Suslin's talks at various conferences and workshops. Each and every lecture of his left me with the greatest admiration for his clarity and elegance of thought, the clean and sharp precision of his words, and the beautiful and inspirational world of mathematics that he created.

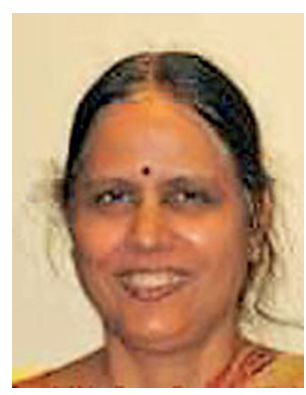

Raman Parimala 


\section{Christophe Soulé}

The first time I met Andrej Suslin was in 1983, when he visited the University Paris 7, invited by Max Karoubi. He made me think of A. Wajda's hero the "iron man." And indeed there was some reason for him to look so strong: since he was a student he had been practicing a painful sport, the building of wooden dachas in the vicinity of Leningrad. He and his team were so well trained that they were able to construct such a dacha in two weeks only!

Suslin had been staying in Paris a couple of months when he made a marvelous discovery: the $K$-theory with coefficients gets unchanged when going from an algebraically closed field to a larger algebraically closed field. To understand why this result was so impressive, one has first to consider the length of its proof: one page only. One has also to consider that till then algebraic $K$-theory was only known for a few rings or for small index (the best being the comparison, due to Merkurjev and Suslin, of $K_{2}$ of a field with the second Galois cohomology group of this field). No result was known till Suslin's breakthrough, which involved general fields and all degrees. This "lightning in the night" gave the hope that $K$-theory and étale cohomology would get compared some day in full generality. And indeed, the notion of "sheaf with transfers," which is used in further work of Suslin and Voevodsky, looks like a grandson of the main argument in Suslin's 1983 paper.

I believe Suslin was very happy during his visit to Paris, both mathematically and privately.

We used to ask him quite often the same question: Do you think that the regime in your country will change some day? And he always answered: This is impossible. Why? Because, for it to change, too many things would have to change.

And again he was right: many things did change.

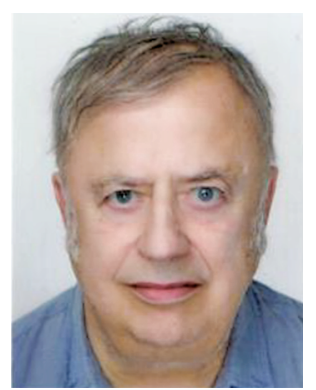

Christophe Soulé

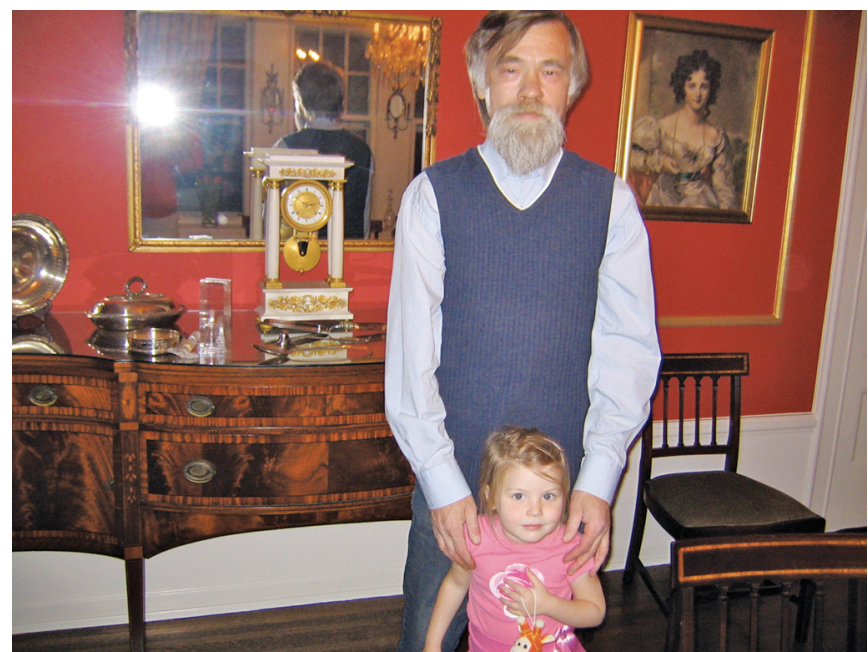

Figure 7. Suslin with his granddaughter, 2011.

\section{Charles Weibel}

Andrei Suslin has been one of the world's most respected mathematicians for the past forty-five years. Luckily for me, his career intersected with mine in many ways, over many decades. I have arranged my memories by decade, since they roughly correspond to the stages of his career.

The 1970s: Serre's conjecture. For much of this decade, the biggest open problem in $K$-theory was called "Serre's conjecture"; it states that all projective modules over a polynomial ring $F\left[x_{1}, \ldots, x_{n}\right]$ are free, where $F$ is a field. At Chicago, where I was a graduate student, any progress was posted on our department's cork bulletin board; Suslin's 1974 thesis (verifying the result for $n=3,4$ ) was announced in this way. In January 1976, we heard that Quillen had established Serre's conjecture in full generality. Because of sporadic communication, we only heard much later that Suslin had also established the result.

I first met Andrei Suslin at the Helsinki ICM in 1978, where he gave an invited lecture about the Serre conjecture. We discovered that he was six weeks younger than I, and that we were both former gymnasts. Sadly, there was no opportunity to talk about math at that time.

The 1980s: Milnor K-theory. In March 1980, Leonid Vaserstein mentioned off-handedly that the Milnor $K$ theory of a field, $K_{n}^{M}(F)$, injects into Quillen's $K$-theory $K_{n}(F)$ modulo torsion, and that Suslin had proven it. This was a complete surprise! That June, there was a $K$-theory conference in Oberwolfach, and Suslin was there, along with Vaserstein, Kato, and many others. One evening, I sat down with Vaserstein and Suslin, and asked Suslin about

Charles Weibel is a professor of mathematics at Rutgers University. His email address is weibe1@math . rutgers . edu.
Christophe Soulé is directeur de recherches au C.N.R.S. at IHÉS. His email address is soule@ihes.fr. 
this surprising assertion. After some discussion between Vaserstein and Suslin, it became clear that Suslin had not proven it, but did have ideas along these lines. The next morning, Andrei came to me with blood-shot eyes, having stayed up all night, and proudly announced: "This theorem-I have proven it!"

In fall 1982, while he was visiting the University of Paris, Suslin amazed us again, settling a special case of the Quillen-Lichtenbaum conjecture by calculating the $K$-theory of algebraically closed fields (with finite coefficients). This became known as "Suslin's rigidity theorem," and was to play a key role in the flood of rapid advances that followed, leading to the first steps towards motivic cohomology for example. Of course, it is easier to prove a theorem once you know it is true; within hours of our hearing this announcement (by word of mouth), I watched as Gillet and Thomason found a slightly different proof over a post-seminar lunch in Princeton.

The 1990s: The Suslin-Voevodsky collaboration. Like many other Russian mathematicians, Suslin came to the US in the early 1990s. I met him briefly in 1991 at the University of Chicago and started receiving email from him in summer 1992, as he began working with a new Harvard PhD named Vladimir Voevodsky. The Suslin-Voevodsky collaboration produced a series of foundational papers on motivic cohomology. Many of us e-collaborated for years in order to digest this material.

I have a vivid memory of Suslin working with a young Voevodsky in 1994 at a Summer School in Hotel Villa Madruzzo, near Trento in Italy. Between Suslin's lectures, they could be spotted at the Villa's fountain, working hard at their mysterious project. It turned out to be a proof that the Bloch-Kato conjecture was equivalent to a conjecture of Beilinson and Lichtenbaum about motivic cohomology.

After Andrei accepted a position at Northwestern University in 1994, I found it hard to contact him; he never used his regular email account and didn't reply to letters. Taking advantage of the fact that my parents lived nearby, I was able to visit him about once a year after that. Since Andrei worked at home and only came in when his classes met, I learned to meet him right after his classes.

The 2000s. By the late 1990s, there were several constructions of a spectral sequence converging from motivic cohomology to the algebraic $K$-theory of a field. In March 1998, at a Great Lakes $K$-theory conference in Notre Dame, Andrei came up to several of us and casually announced that these spectral sequences could be extended from fields to varieties. This observation has been very useful in my subsequent research.

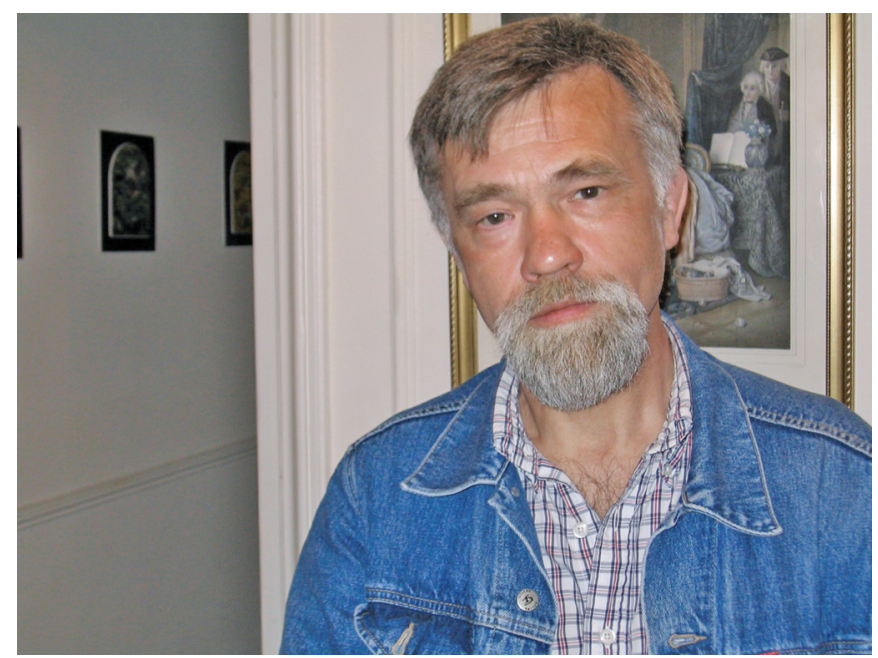

Figure 8. In Paris around 2005.

In 2004-05, Suslin and Rost were at the Institute for Advanced Study in Princeton as part of a special year organized by Voevodsky. Voevodsky had just released a preprint claiming to prove the Bloch-Kato/BeilinsonLichtenbaum conjectures, modulo the existence of certain varieties, now called Rost varieties. Suslin's lectures in fall 2004, and those of Markus Rost in the spring, gave a proof that these varieties existed. This proof was carefully written and published by Haesemeyer and me in 2007.

By 2007-08, Andrei told me that he was looking for a new problem to solve. I last saw Andrei in 2013 at a conference at USC. After that, I believe he spent most of his summers in St. Petersburg with his family; my main communication with him was through Eric Friedlander. He last taught at Northwestern in spring 2017.

I will miss him, and I'm sure I'm not alone in that.

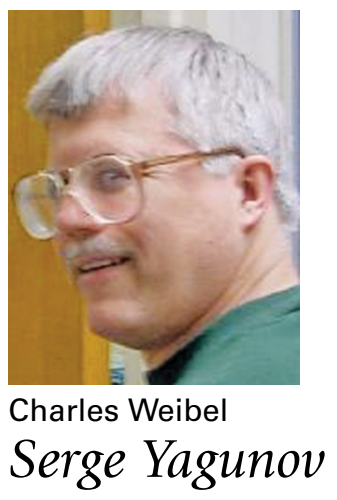

As the first student of Andrei at Northwestern University as well as his graduate student in St. Petersburg, I had the amazing opportunity to communicate with him both in

Serge Yagunov is a senior research fellow at the St. Petersburg Department of Steklov Institute of Mathematics. His email address is yagunov@gmai 1 . com. 
St. Petersburg and in Evanston. For several years our paths intersected in different countries, and the conversations and chats we had at that time left a deep mark on my professional life and in my heart.

The first time I met Andrei Suslin was in Peterhof in February 1988, where Andrei was running a seminar on commutative algebra. Prior to the seminar, the students had already heard a lot about him. Andrei was well known as an International Mathematical Olympiad gold medal winner, one of the strongest mathematicians in St. Petersburg, the youngest doctor of sciences ${ }^{1}$ in the USSR, and finally as a plenary speaker at the ICM 86 in Berkeley who could not attend the Congress for political reasons. Certainly, it was a very exciting opportunity to meet him in person.

Andrei employed a very informal and charming style of lecturing in the seminar. His explanations seemed very clear-they only required translation into the standard language of mathematics. However, this last step was absolutely not easy, insofar as it required a strong mathematical background from the seminar attendees. The exams for the class felt like a conversation among colleagues discussing a problem.

In 1993, Andrei became my thesis advisor despite his frequent travels abroad. At times when he would return to St. Petersburg, Andrei would generously share with me his ideas and approaches to problems. Soon after I joined Andrei at Northwestern in the fall of 1994, I made a mathematical breakthrough. Andrei's comment "Oh, it is beautiful" is praise I shall always value.

The local algebraic activity in St. Petersburg was also galvanized by Andrei's presence. This mostly took the form of long evening discussions at Steklov Institute. These usually started at about 5 or $6 \mathrm{pm}$ and often continued until the porter came to close the institute for the night. Besides Andrei, the participants included Alexander Merkurjev, Ivan Panin, and Oleg Izhbolodin (who prematurely died in 2000). The topics discussed at that time mostly were higher Chow groups and étale cohomology. This, probably, was an introductory step to Andrei's joint work with Vladimir Voevodsky on the Milnor conjecture. These evening conversations played an important role in creating the current St. Petersburg school of algebraic geometry and $K$-theory. Now almost every algebraist in St. Petersburg who is younger than sixty is either a coauthor of Andrei, his former student, or one of his mathematical descendents.
I vividly remember Andrei smoking on the porch of Lunt Hall-the only place where smoking was allowed by university regulations. I always tried to catch him there, since it was a good chance to discuss mathematics or just chat without disturbing him too much in his office.

Thank you, Andrei!

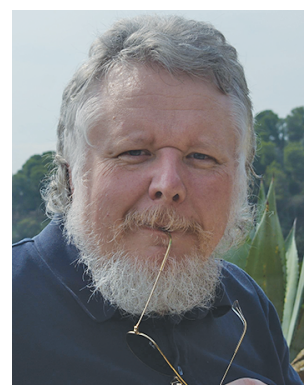

Serge Yagunov

\section{Credits}

Figure 1 is courtesy of Olga Suslina.

Figures 2, 6, and 8 are courtesy of Eric Friedlander.

Figure 3 is courtesy of Alexander Merkurjev.

Figures 4 and 5 are courtesy of Eugene Potkin.

Figure 7 is courtesy of Maria Suslina.

Photo of Alexander Beilinson is courtesy of Irene Ogievetskaya.

Photo of Eric M. Friedlander is courtesy of Susan Friedlander. Photo of Christian Haesemeyer is courtesy of Christian Haesemeyer.

Photo of Marc Levine is courtesy of University of DuisburgEssen.

Photo of Alexander Merkurjev is courtesy of Olga Merkurjev. Photo of Ivan Panin is courtesy of Ivan Panin.

Photo of Raman Parimala is courtesy of Skip Garibaldi.

Photo of Christophe Soulé is courtesy of Studio Maubeuge. Photo of Charles Weibel is courtesy of Charles Weibel. Photo of Serge Yagunov is courtesy of Victoria Shpacovitch.

\footnotetext{
${ }^{1}$ A degree beyond the PhD similar to the German Habilitation.
} 